\title{
Fractured phalanx in a violinist's finger: Special considerations in treating musician patients
}

\author{
Julia Gibson ${ }^{1}$, Jason Williams² \\ ${ }^{1}$ Class of 2012, Faculty of Medicine, Dalhousie University \\ ${ }^{2}$ Department of Surgery, Division of Plastic and Reconstructive Surgery, Dalhousie University
}

\begin{abstract}
Post-traumatic reconstructive surgery on musician patients is uniquely challenging in that sustained motor deficits imperceptible to the average person can be debilitating for this population. ${ }^{1}$ Instrument manipulation is extremely physically demanding; even without trauma musicians are at risk of developing a musculoskeletal disorder from mere overuse of the muscles involved in their craft. ${ }^{2}$ Furthermore the link between physical and emotional well-being is exaggerated in musicians, as their livelihood revolves around the physical expression of emotion. ${ }^{3}$ Analyzing medical treatment from a musician's perspective provides a useful demonstration of the increased efficacy in utilizing a whole person approach as opposed to one that is strictly biomedical. ${ }^{4}$ Heightened sensitivity to motor deficits and the economic and emotional implications thereof are significant factors pertaining to the lifestyle of these patients, and treatment should be tailored accordingly. ${ }^{5}$
\end{abstract}

\section{Case Presentation}

Tn February 2007, a 52-year-old violinist and music teacher opened a steel-framed classroom window, which then fell and crushed her left index finger. Being her string-pressing hand, the impact of this injury was potentially devastating. Unfortunately, she did sustain an oblique fracture of her proximal phalanx, which was near the proximal interphalangeal (PIP) joint but was not interarticular (Figure 1). Additionally, there was a laceration on the volar aspect of the same finger.

Three days following her injury, she was brought to the operating room for definitive repair. Previously placed sutures were removed, and the incision was extended by $1 \mathrm{~cm}$ on the radial aspect in line with the PIP joint. Careful dissection revealed the tendon sheath and digital nerve on the radial aspect to be intact, though the latter appeared to have sustained some damage. The location of the fracture between opposing flexor and extensor tendons caused displacement of the bone fragments rendering the injury inherently unstable. However, once the fracture planes were exposed, reduction was easily achieved and held using termite forceps. Fixation was then attained using two percutaneous 0.035 inch K-wires. These traversed the fracture from the distal portion of the proximal phalanx into the proximal cortex dorsoventrally. The result, as visualized on a portable x-ray machine (Figure 2), was an excellent reduction, and the wires were trimmed near skin level. The skin edges, which were somewhat macerated, were then debrided and closed using 5-0 Prolene sutures. The final step of the procedure was application of Jelonet, gauze and a volar splint.

\section{Discussion}

\section{Rehabilitation}

The exercise best suited to target the precise fine motor movements required to manipulate an instrument is to play the instrument itself. Avoiding prolonged periods of immobility is thus critical towards rehabilitating injured regions, as well as maintaining function in uninjured tissues. ${ }^{6}$ Along with improving physical rehabilitation, an early return to playing is also a key factor in protecting the mental state of injured musicians, who are at risk of becoming depressed if unable to play. ${ }^{7}$ In circumstances requiring the shortest possible post-operative immobilization period, open reduction and internal fixation procedures should be used even when external fixation would provide successful repair in the average patient. ${ }^{6}$ This treatment also better enables the surgeon to preclude rotational deformities, which are intolerable even at deviations as minute as $5^{\circ}$ in string pressing fingers. ${ }^{8}$

Recognizing the significance of the patient's career, her post-operative hand therapy was focused towards enabling her to resume playing the violin promptly. As she had sustained significant trauma, however, this progression was gradual in order to avoid secondary musculoskeletal problems. ${ }^{1}$ For this reason, she was advised against attempting to play her instrument for the first three weeks following the surgery to allow fracture healing to commence. The early stages of her rehabilitation involved simple flexion-extension exercises for the digits of her injured hand. Two balls, offering different degrees of resistance, were given to her to squeeze. Once she had regained the strength necessary to compress the softer of the two, she progressed to squeezing the firmer. 


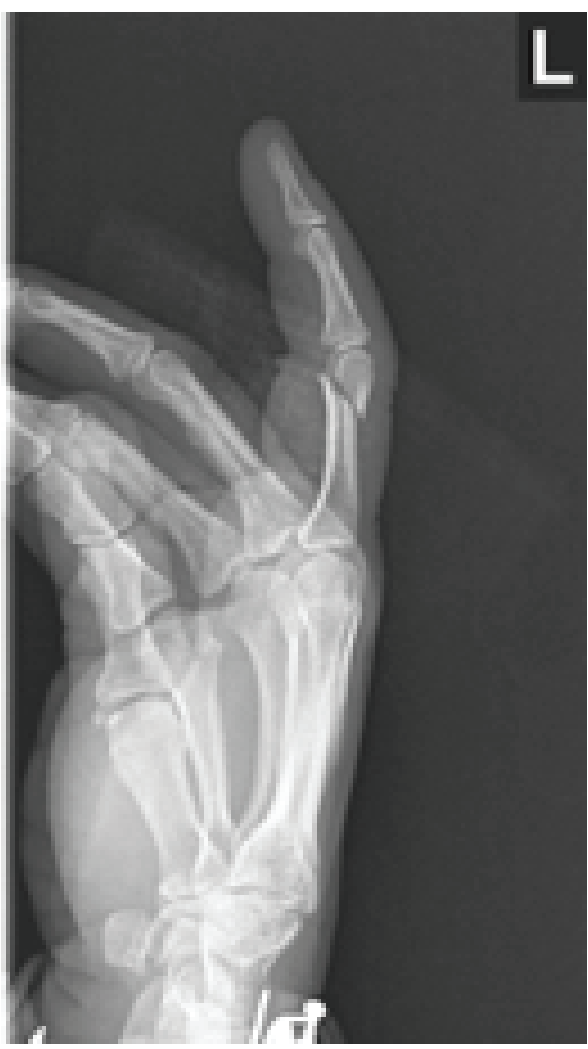

Figure 1. Pre-operational X-ray of fractured proximal phalange.

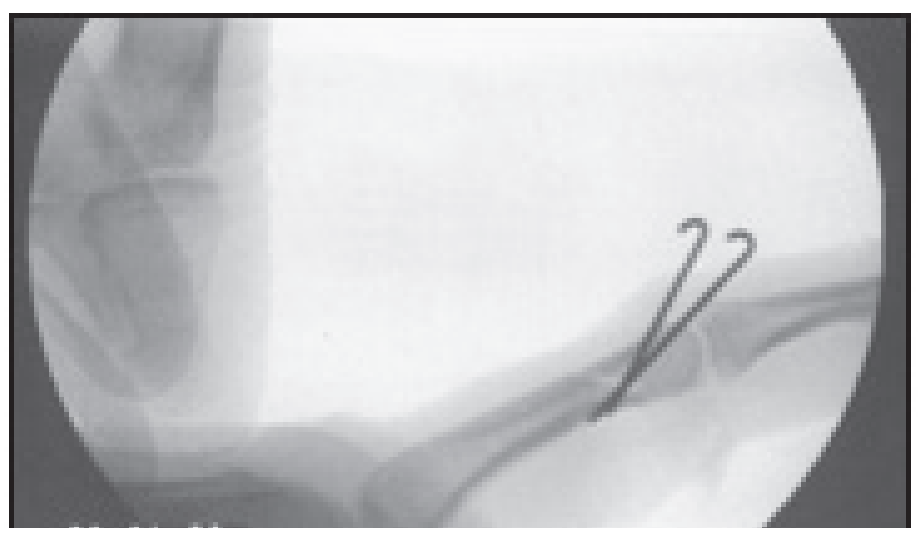

Figure 2. Fixation of fracture in proximal index phalange using two K-wires.

She was eager to diligently perform her exercises but was cautioned by the occupational therapist to not overwork her hand, as doing so could impede recovery and worsen the post-operative inflammation. She was also advised to gently massage the injured area throughout the rehabilitation period in order to minimize swelling.

Limited "shadow playing" exercises provide a safe and effective means of incorporating an instrument into a rehabilitation program even while the injured finger is not sufficiently healed to perform. ${ }^{9}$ Initially these exercises only involved the positioning of the patient's fingers on the instrument. Gradually, position shifts were followed by attempts to lightly press the strings. The force applied to the strings increased incrementally as her strength returned. Utilizing the violin in early rehabilitation stages not only strengthens injured tissues, but also assists in sensory re-education. ${ }^{6}$ The patient initially reported a sensory deficit in the distal portion of her finger, and hypersensitivity of the skin surrounding the incision site. She experienced pain each time the hypersensitive region made contact with the violin, and especially when it became caught between the strings. To prevent this irritation, the occupational therapist provided a silicone sleeve to surround the injured finger. The sleeve was found to be effective while in place, but would often come loose and get in the way.

Musicians typically adhere to post-operative rehabilitation programs with a degree of discipline and motivation which notably surpasses that of the average patient. ${ }^{1}$ Our patient was no exception, and within four months she was informed by her occupational therapist that the injured finger had regained complete "functional mobility." Although it could not meet the exigent demands of a professional violinist's repertoire, she was able to flex and extend the digit enough to fully accommodate activities of daily living, and sensation was returning to the distal portion. Nine months after surgery, the inflammation was barely perceptible, and she no longer experienced pain when the scar tissue came in contact with the violin. Although the scar tissue had softened considerably, it remained as a raised, irregular surface on the underside of her finger. Of note, the option to surgically revise this in the future was considered.

Continued diligence towards practicing the violin has resulted in ever-improving levels of strength and dexterity in her injured finger. Nine months following the operation she was able to play medium-paced music for extended periods of time without discomfort. This, along with her regained ability to position her fingers with ease, has enabled her to continue her career as a music teacher. The pieces she teaches to her elementary students are not overly demanding, however, more challenging pieces prove problematic, as her finger has not yet fully regained its former level of motor functioning. During the more advanced violin arrangements, she develops a burning discomfort in the base of her finger if she plays for upwards of fifteen minutes. She also reported experiencing intense, sharp pain originating at the distal phalange and radiating proximally when attempting the prolonged tension required for optimal intonation. Intonation is a measure of accuracy of pitch in the produced sound, and is thus of the utmost importance to musicians. ${ }^{10}$ While teaching, the patient noted an absence of such discomfort when performing on the piano, no matter how fast or lengthy the piece.

Even for the fittest hand, the violin is a particularly physically challenging instrument. Research has shown 
that among musicians, playing a stringed instrument and being female appear to be two of the biggest risk factors for developing a playing-related musculoskeletal disorder. ${ }^{11}$ The string-pressing hand of a violinist is constantly challenged to perform frequent shifts in position and to depress strings with enough force to produce the desired intonation (Figures 3 and 4). ${ }^{12}$ The present patient has attempted to compensate for her deficit in these areas by releasing her index finger between position shifts, and by substituting her middle finger where possible. While this effectively decreases the strain on her injured index finger, she reports a resulting decline in intonation. Though this decrease in quality of sound is imperceptible to the average person's ear, it is glaringly obvious to her. She has also made postural adjustments to render prolonged flexion less painful. These involve hunching the left shoulder downward and curling the left wrist forward, further around the neck of the instrument. These compensatory mechanisms offer limited alleviation of her discomfort, but are problematic. Improper posture and sudden changes in playing technique can be physically straining and cause playing-related injuries. ${ }^{2}$ Furthermore, the musician has had extensive professional training, which renders the obligation to compromise technique a frustrating experience indeed.

\section{Patient's perspective}

For many musicians, playing music not only enables them to make a living, but also gives them a passion for life itself. ${ }^{5}$ This is true of the current patient who finds her career as a music teacher professionally rewarding, but also views the violin as her primary form of creative expression, and thus central to her identity. Having played since the age of six, she had mastered the skills required to play this challenging instrument to its fullest. This former proficiency is what renders her current physical impairments extremely frustrating and upsetting. Sensitivity to these factors is crucial when treating musicians. ${ }^{1}$ Initially, the thought of requiring surgery on her finger caused extreme anxiety for her. Health professionals were able to assuage this by expressing their appreciation for how critical adequate restoration of function was to her as a musician. This strengthened the patient's confidence in both the proposed surgical treatment as well as the suggested rehabilitation exercises. Her first post-operative encounter with the violin, however, has remained a vivid and painful memory. The physical discomfort associated with this experience was heavily outweighed by the emotional anguish it caused her. She reported feeling "absolutely miserable" because the activity that was once her most perfected skill and treasured means of expression was at that point completely lost to her. Along with having concerns about the future of her music teaching career, she experienced thoughts such as "Why did

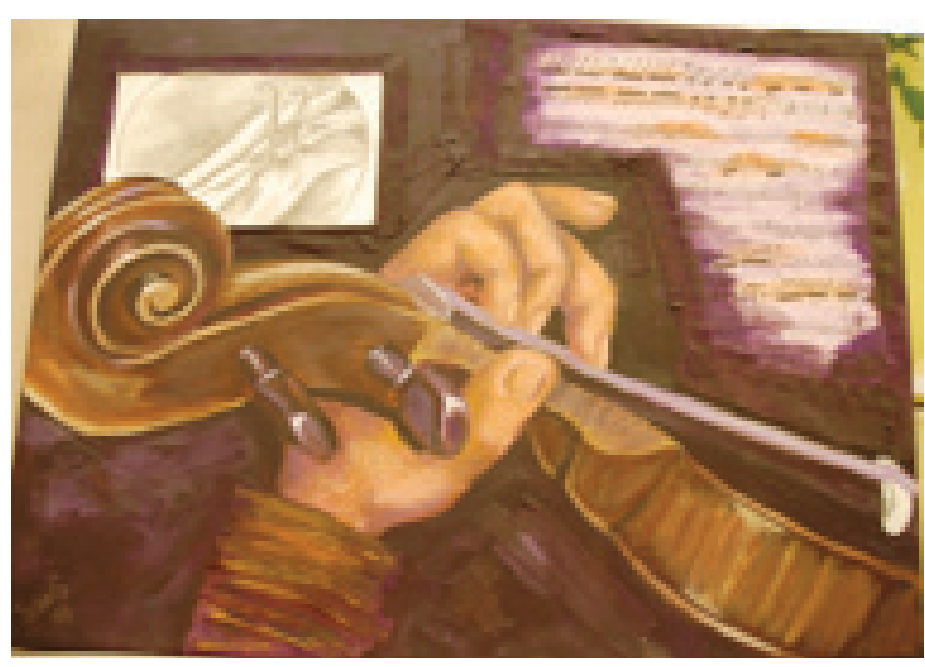

Figure 3. Author's depiction of post-operative posture and X-ray image.

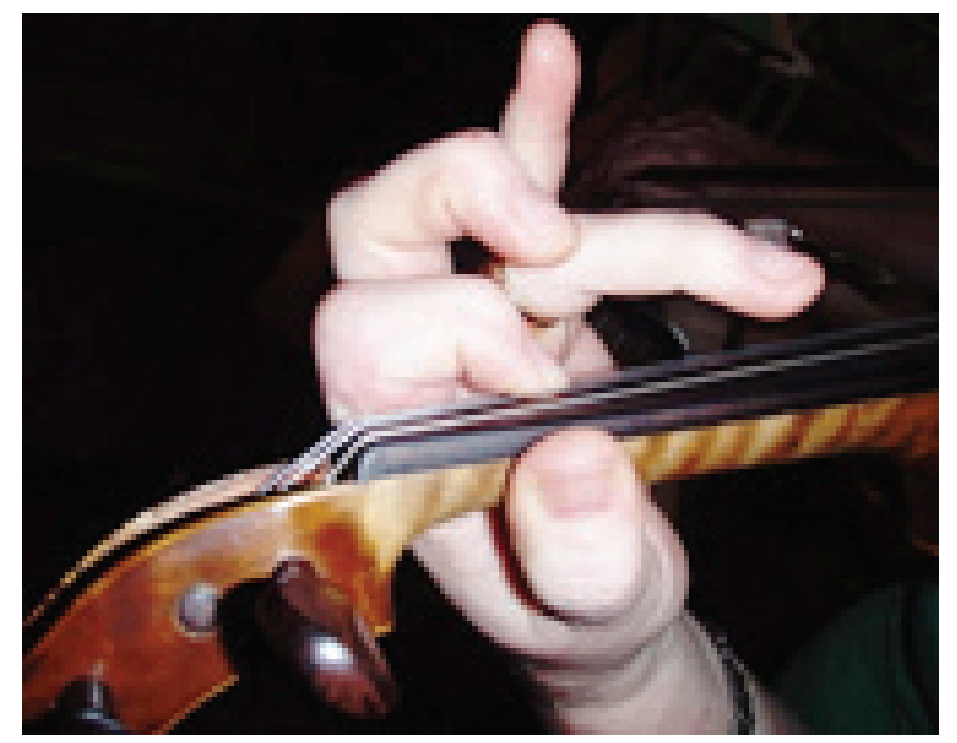

Figure 4. Post-operation pressing of string with index finger.

this happen to me?" and "I want my life back somehow," reflecting the severe emotional toll this injury took on her.

Luckily, she decided to channel her frustration and anxiety into motivation towards regaining function, and put every effort towards successful rehabilitation. She was pleased with her finger's eventual functional rehabilitation in terms of everyday living and moderate violin playing, but reported a sense of disappointment in herself when attempting the more challenging pieces. As performing such pieces used to be a major source of personal satisfaction and joy for her, she remains hopeful that her finger will regain its prior level of extraordinary fitness. Her continued improvements in motor function and sensation are encouraging. She expressed her belief that had the surgeon and occupational therapist not successfully addressed her specific physical needs and concerns as a musician, rehabilitation would have been more frustrating and less successful. 


\section{Conclusion}

The case of a skilled violinist who suffered a fractured proximal phalange in her string-pressing hand demonstrates that musicians can have more exigent demands than the average patient in terms of rehabilitation. After undergoing open reduction and pin fixation of the fracture with extensive rehabilitation, her finger was restored to a state which would be considered completely functional in the average patient. She, however, remained frustrated with its performance, as it could not meet the stringent demands she was accustomed to placing upon it when playing the violin. Additionally, she reported sadness and frustration as a result of her reduced capacity to play. Her experience shows that such injuries affect not only the physical well-being of musician patients, but also their livelihood and psychological well-being. This case thus provides an effective demonstration of the importance of a holistic approach to treatment within the realm of reconstructive surgery despite the intensely physical nature of the procedures performed therein. By adjusting to her specific needs as a musician, treatment was more effective and successful for this patient.

\section{REFERENCES}

1. Winspur I. Special operative considerations in musicians. Hand Clin 2003;19:247-258.

2. Brandfonbrener AG. Musculoskeletal problems of instrumental musicians. Hand Clin 2003;19(2):231-9.

3. Wynn Parry CB. Introduction: The musician's hand. Hand Clin 2003;19:211213.

4. Salter M, Cheshire L. Hand Therapy: Principles and Practice. Oxford: Butterworth Heinemann, 2000:ix.

5. Winspur I, Wynn Parry CB. The Musician's Hand: A Clinical Guide. London: Martin Dunitz Ltd, 1998:48-52.

6. Warrington J. Hand therapy for the musician: Instrument-focused rehabilitation. Hand Clin 2003; 19:287-301.

7. Wynn Parry CB. The Musician's Hand. In: Salter M, Cheshire L, ed. Hand Therapy—Principles and Practice. Oxford: Butterworth Heinemann; 2000. p. 226-36.

8. Winspur I. Advances in objective assessment of hand function and outcome: Assessment of the musician's hand. Hand Clin 2003;19:483-493.

9. Winspur I, Wynn Parry C. Musicians' hands: A surgeon's perspective. Med Prob Performing Artists 2000;15(1):31-4.

10. The Harvard Dictionary of Music. Cambridge: Harvard University Press, 2003. s.v. "Intonation",

11. Zaza C, Farewell VT. Musicians' playing-related musculoskeletal disorders: An examination of risk factors. Am J Ind Med 1997;32:292-300.

12. Blum J. Examination and interface with the musician. Hand Clin 2003;19:223230.
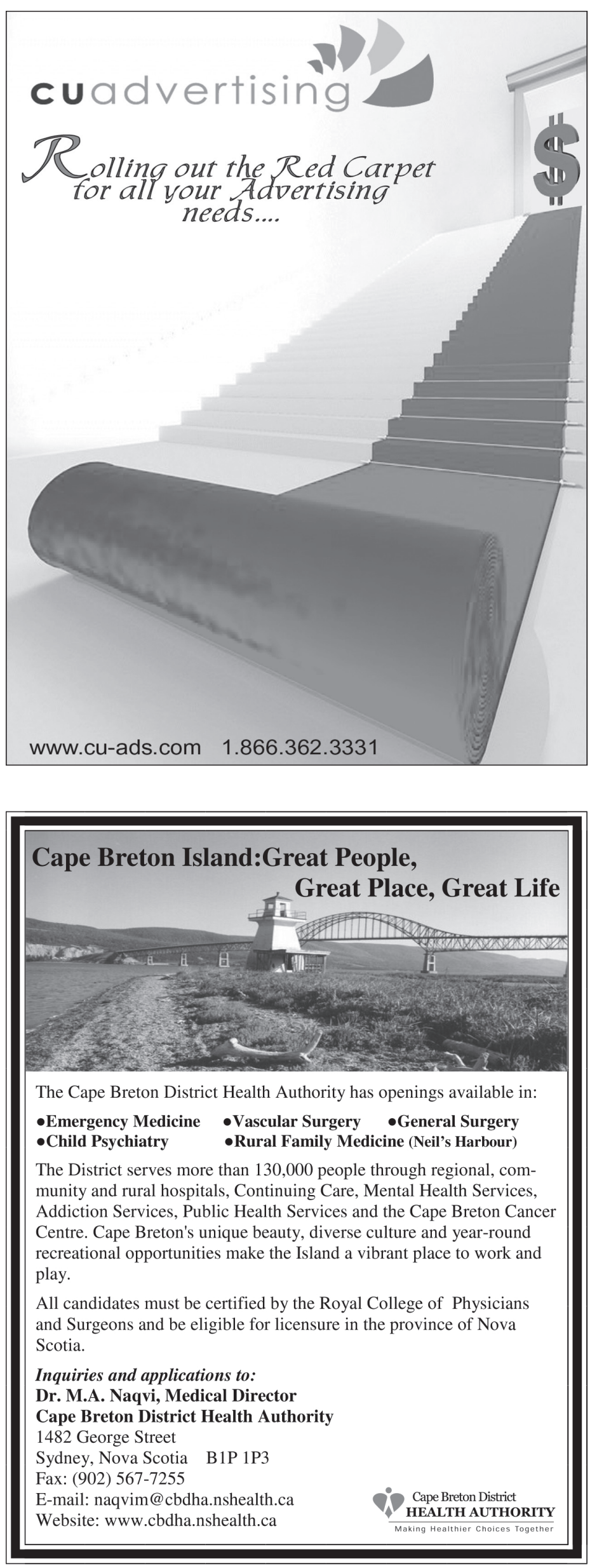